feldes $B$. Aus (50) folgt dann für das Beweglichkeitsverhältnis

$$
\frac{\mu(0)}{\mu(B)}=\frac{r_{\square}^{2}(B)}{r_{\odot}(B)} .
$$

In Abb. 12 sind die Ergebnisse der numerischen Auswertung ${ }^{11}$ des Integralausdrucks (22) mit (23) und (24) für den Widerstand rechteckförmiger Proben in einer Kurvenschar zusammengestellt. Aufgetragen wurde der Geometriefaktor $\sigma(B) / \sigma(0) \cdot r(B)$ über $\operatorname{tg} \Theta$. Kurvenparameter ist das Seitenverhältnis $a / b$. Für kleine HaLL-Winkel $\Theta$ verlaufen alle Kurven quadratisch in $\Theta$, während für $\operatorname{tg} \Theta \gg 1$ das bereits diskutierte lineare Verhalten in $\operatorname{tg} \Theta$ erkennbar ist. Die in Abb. 12 zusammengefaßten numerischen Auswertungen bilden eine Abrundung der theoretischen Behandlung des Geometrieeinflusses auf den transversalen Widerstandseffekt rechteckförmiger Halbleiterplatten. Sie erschließen quantitativ den durch die hergeleiteten analytischen Entwicklungsausdrücke nicht erfaßten Übergangsbereich zwischen kleinen und großen HALL-Winkeln $\Theta$ in Abb. 9.

Die gewonnenen theoretischen Ergebnisse stehen in quantitativer Übereinstimmung mit den Messungen des Widerstandseffektes an rechteckförmigen Proben aus Indiumantimonid nach $\mathrm{W}_{\text {EISS }}$ und WELKER ${ }^{2}$.

11 Für die numerische Auswertung der Integrale (23) und (24) danken wir den Herren H. Hofrmans und G. Zegin

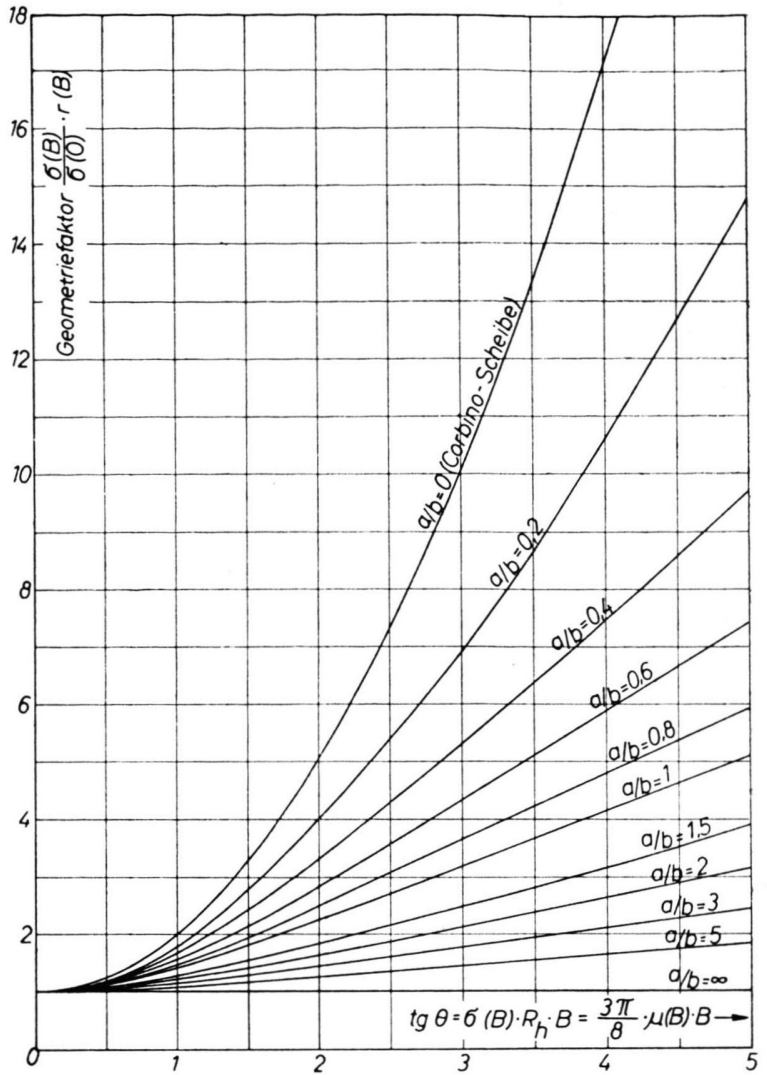

Abb. 12. Geometriefaktor $\sigma(B) / \sigma(0) \cdot r(B)$ rechteckiger Halbleiterplatten als Funktion von $\operatorname{tg} \Theta$ mit $a / b$ als Parameter.

vom Mathematischen Institut der Siemens-Schuckertwerke AG, Erlangen.

\title{
Der Geometrieeinfluß auf den Hall-Effekt bei rechteckigen Halbleiterplatten
}

\section{Von Hans-Joachim Lippmann und Friedrich Kuhrt}

Aus dem Laboratorium der Zentralwerksverwaltung der Siemens-Schuckertwerke AG, Nürnberg (Z. Naturforschg. 13 a, $474-483$ [1958] ; eingegangen am 20. März 1958)

Die HaLl-Spannung einer rechteckigen, stromdurchflossenen Halbleiterplatte im transversalen Magnetfeld wird durch Lösung des Potentialproblems berechnet. Der sich für die Halt-Spannung ergebende Ausdruck ist das Produkt aus der Hall-Spannung der unendlich langgestreckten Platte und einer Geometriefunktion, die vom Seitenverhältnis $a / b$ des Rechtecks und dem HALL-Winkel $\Theta$ abhängt. Für kleine und große $\mathrm{H}_{\mathrm{ALL}}$-Winkel werden analytische Ausdrücke für die Geometriefunktion abgeleitet, während das Übergangsgebiet zwischen kleinen und großen $\Theta$-Werten durch numerische Auswertung der in der Geometriefunktion auftretenden Integrale erschlossen wird.

Der bekannte Ausdruck für die Hall-Spannung eines Elektronenleiters im transversalen Magnetfeld $B$

$$
U_{\mathrm{h} \infty}=\frac{R_{\mathrm{h}}}{d} i_{\mathrm{S}} B
$$

gilt nur für unendlich langgestreckte Platten. Hierin ist $R_{\mathrm{h}}$ die HaLL-Konstante des Elektronenleiters, $d$ die Dicke der Platte und $i_{\mathrm{s}}$ der die Probe durchfließende Steuerstrom. Bei rechteckigen Halbleiter- 
platten endlicher Seitenabmessungen $a$ und $b$ nach Abb. 1 tritt in der Mitte der Platte zwischen den Punkten $P$ und $Q$ eine Hall-Spannung $U_{\mathrm{h}}$ auf, die nicht mehr durch Gl. (1) beschrieben wird ${ }^{1,2,3,4}$.

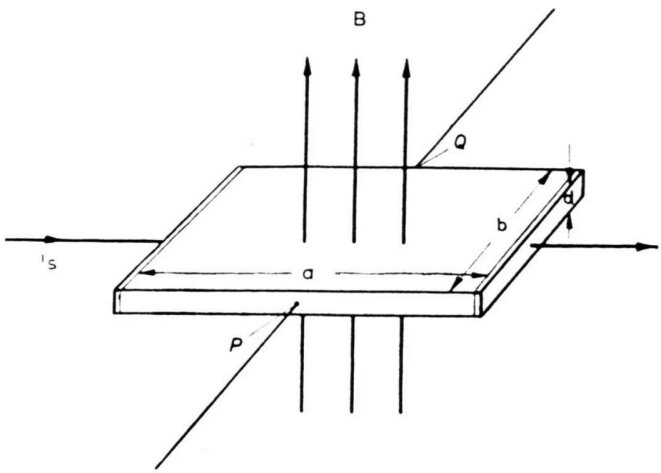

Abb. 1. Rechteckige, stromdurchflossene Halbleiterplatte im transversalen Magnetfeld $B$.

Die Ableitung von Gl. (1) beruht auf der wesentlichen Voraussetzung, daß zwischen den beiden punktförmigen HALL-Elektroden $P$ und $Q$ der Stromdichtevektor nur eine Komponente in Längsrichtung der Platte besitzt. In einer rechteckigen Platte mit endlichem Seitenverhältnis $a / b$, bei der sich die metallisch leitenden Stromelektroden (hohe Leitfähigkeit bei kleiner Elektronenbeweglichkeit) über die gesamte Breite $b$ der Platte erstrecken, werden die Strombahnen durch das Magnetfeld seitlich abgedrängt ${ }^{5,6}$. Der Stromdichtevektor bekommt dadurch eine Querkomponente, so daß die Voraussetzung für die Gültigkeit von Gl. (1) nicht mehr erfüllt ist.

Ausgehend von der Lösung des Potentialproblems einer stromdurchflossenen Platte im transversalen Magnetfeld ${ }^{3,6}$ wird im folgenden die in der Mitte einer rechteckigen Halbleiterplatte mit den Seitenabmessungen $a$ und $b$ auftretende HALL-Spannung $U_{\mathrm{h}}$ berechnet. In die Berechnung gehen als elektrische Eigenschaften des Halbleitermaterials HaLL-Konstante $R_{\mathrm{h}}$ und Leitfähigkeit $\sigma$ ein, wobei $\sigma$ wegen des physikalischen Widerstandseffektes als Funktion von $B$, also $\sigma=\sigma(B)$ gegeben ist.

\section{Grundlagen}

Die rechteckige Halbleiterplatte mit den Seiten $a$ und $b$ und der Dicke $d$ liege parallel zur $x y$-Ebene

1 J. Isenberg, B. R. Russell u. R. F. Greene, Rev. Sci. Instrum. 19 (Nr. 10), 685 [1948].

2 J. Volger, Phys. Rev. 79 (Nr. 6), 1023 [1950]. gemäß Abb. 2. Das angelegte homogene Magnetfeld soll senkrecht auf der Plattenebene stehen ( $z$-Richtung). Dann sind die elektrische Feldstärke $\mathbb{F}$ und die Stromdichte j parallel zur $x y$-Ebene und nur

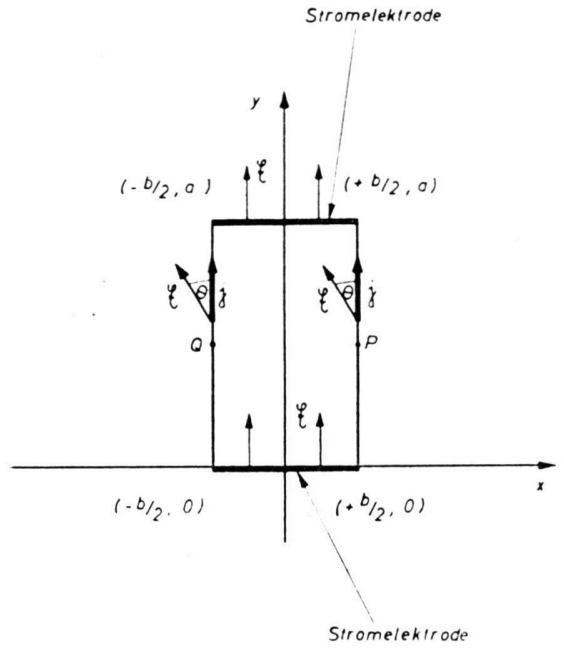

Abb. 2. Verhalten von $\mathfrak{E}$ und $\mathrm{j}$ auf den Rändern einer mit Elektroden versehenen rechteckigen Halbleiterplatte.

noch Funktionen von $x$ und $y$ (zweidimensionales Problem).

Das elektrische Feld $\mathfrak{F}$ ist ein Potentialfeld. Für das elektrische Potential $\varphi(x, y)$ gilt daher

$$
\Delta \varphi(x, y)=0 .
$$

Zur Bestimmung der Potentialfunktion $\varphi(x, y) \mathrm{mu}$ die Differentialgl. (2) unter folgenden Randbedingungen gelöst werden: $\mathrm{Da}$ die Stromelektroden Äquipotentiallinien sind, muß auf den $b$-Rändern gelten

$$
\varphi(x, 0)=U \quad \text { und } \quad \varphi(x, a)=0 .
$$

$U$ ist die Spannung an der Platte. Der Hald-Winkel $\Theta$ zwischen der elektrischen Feldstärke $\leftarrow$ und der Stromdichte j definiert durch

$$
\operatorname{tg} \Theta=\sigma(B) R_{\mathrm{h}} B
$$

ist bei homogenem Magnetfeld $B$ eine Invariante. Die Randbedingungen für die Potentialfunktion $\varphi(x, y)$ auf den beiden $a$-Rändern (Abb. 2) lauten daher

$$
-\left(\frac{\partial \varphi}{\partial x} / \frac{\partial \varphi}{\partial y}\right)_{x= \pm b / 2}=\sigma(B) R_{\mathrm{h}} B
$$

3 R. F. W Іск, J. Appl. Phys. 25 (Nr. 6), 741 [1954].

4 F. Kuнrt, Eigenschaften der Hall-Generatoren, SiemensZeitschrift, S. 370-376 [1954]. 


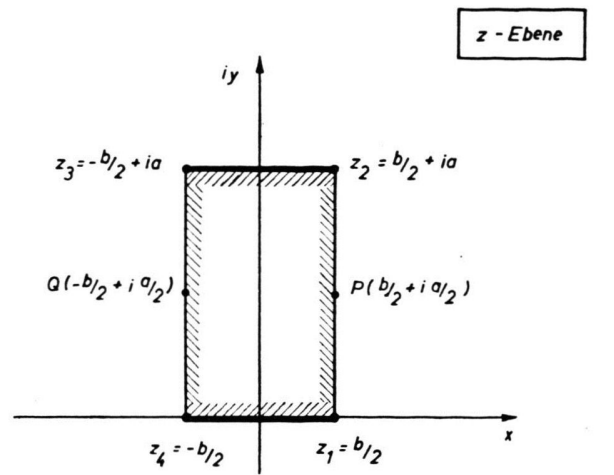

Abb. 3 a.

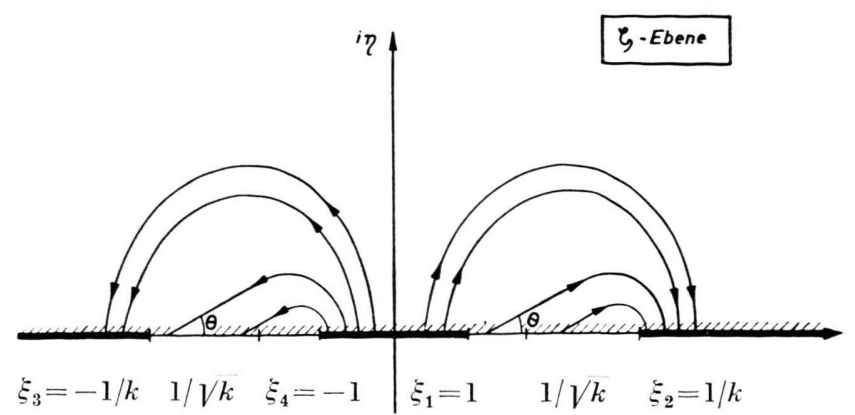

Abb. 3 b.

Abb. 3. Abbildung des Rechtecks mit den Seiten $a$ und $b$ auf die obere $\zeta$-Halbebene mit schematischem Verlauf der elektrischen Feldlinien in der $\zeta$-Ebene.

Die Lösung der Differentialgl. (2) mit den Randbedingungen (3) und (5) erfolgt durch konforme Abbildung. $\mathrm{Zu}$ diesem Zweck wird die $x y$-Ebene (Abb. 2) als komplexe $z=(x+i y)$-Ebene (Abb. 3a) aufgefaßt und die Abbildung des Inneren des Rechtecks auf die obere Hälfte einer komplexen $\zeta$-Ebene (Abb. 3 b) betrachtet. Diese Abbildung erfolgt durch die Funktion ${ }^{6}$

$$
z(\zeta)=\frac{b}{2 K(k)} \int_{0}^{\zeta} \frac{\mathrm{d} \zeta}{\sqrt{\left(1-\zeta^{2}\right)\left(1-k^{2} \zeta^{2}\right)}}
$$

mit $K(k)$ als vollständiges elliptisches Integral erster Gattung als Funktion des Moduls $k$. Durch die Abbildungsfunktion (6) wird der Rand des Rechtecks in die reelle Achse ( $\xi$-Achse) der $\zeta$-Ebene übergeführt, wobei die Eckpunkte $z_{1}=b / 2 ; z_{2}=b / 2+i a$; $z_{3}=-b / 2+i a$ und $z_{4}=-b / 2$ in die Punkte $\xi_{1}=+1 ; \xi_{2}=+1 / k ; \xi_{3}=-1 / k$ und $\xi_{4}=-1$ übergehen. Die punktförmigen Hall-Elektroden $P$ und $Q$, d. h. die Punkte $(b / 2+i a / 2)$ und $(-b / 2+i a / 2)$ in $\operatorname{der} z$-Ebene werden auf die Punkte $+1 / \sqrt{k}$ und $-1 / \sqrt{k}$ in der $\zeta$-Ebene abgebildet. Der Modul $k$ ist durch das Seitenverhältnis $a / b$ über die transzendente Gleichung

$$
\frac{a}{b}=\frac{K\left(\sqrt{1-k^{2}}\right)}{2 K(k)}
$$

bestimmt. Abb. 4 zeigt den durch (7) beschriebenen Zusammenhang zwischen $a / b$ und $k$. Für kleine $a / b$ und für große $a / b$ können für Gl. (7) unter Ver-

${ }^{5}$ H. WeIsS u. H. Welker, Z. Phys. 138, 322 [1954].

${ }^{6}$ H. J. Lippmann u. F. Kuhrt, Z. Naturforschg. 13 a, 464 [1958], voranstehende Arbeit. wendung der Entwicklungen ${ }^{7}$ der vollständigen elliptischen Integrale $K(k)$ und $K\left(\sqrt{ } 1-k^{2}\right)$ einfache Näherungsausdrücke angegeben werden. Sie lauten

$$
k=1-8 \exp \left\{-\frac{\pi}{2} \frac{b}{a}\right\}
$$

für den Bereich $0 \leq a / b \leq 0,35$

und

$$
k=4 \exp \left\{-\pi \frac{a}{b}\right\}
$$

für den Bereich $\quad 0,85 \leq a / b \leq \infty$

und beschreiben in den angegebenen Bereichen Gl. (7) mit einer Genauigkeit von ca. $1 \%$.

Bei der konformen Abbildung nach Gl. (6) geht das elektrische Potential $\varphi(x, y)$ über in eine Potentialfunktion $v(\xi, \eta)$ in der $\zeta$-Ebene. Für $v(\xi, \eta)$ gelten entsprechende Randbedingungen auf der $\xi$-Achse wie für $\varphi(x, y)$ auf den Rändern des Rechtecks in der $z$-Ebene. Die elektrischen Feldlinien haben in der oberen $\zeta$-Halbebene den in Abb. $3 \mathrm{~b}$ dargestellten qualitativen Verlauf. Die durch die Potentialfunktion $v(\xi, \eta)$ bestimmte Feldstärke $\mathfrak{F}(\xi, \eta)$ kann explizit angegeben werden (vgl. Anm. ${ }^{3,6}$ )

$$
\begin{aligned}
& |\xi(\xi, \eta)| \\
& =\frac{C^{\prime}}{|(\zeta-1)(\zeta+[1 / k])| 1 / 2-\Theta / \pi \mid(\zeta+1)(\zeta-[1 / k])^{1 / 2+\Theta / \pi}} ;
\end{aligned}
$$

$C^{\prime}$ ist eine Konstante, die im folgenden Abschnitt bei der Berechnung der Hall-Spannung angegeben wird.

7 F. Oberhettinger u. W. Magnus, Anwendung der elliptischen Funktionen in Physik und Technik, Springer-Verlag, Berlin/Göttingen/Heidelberg 1949, S. 3. 


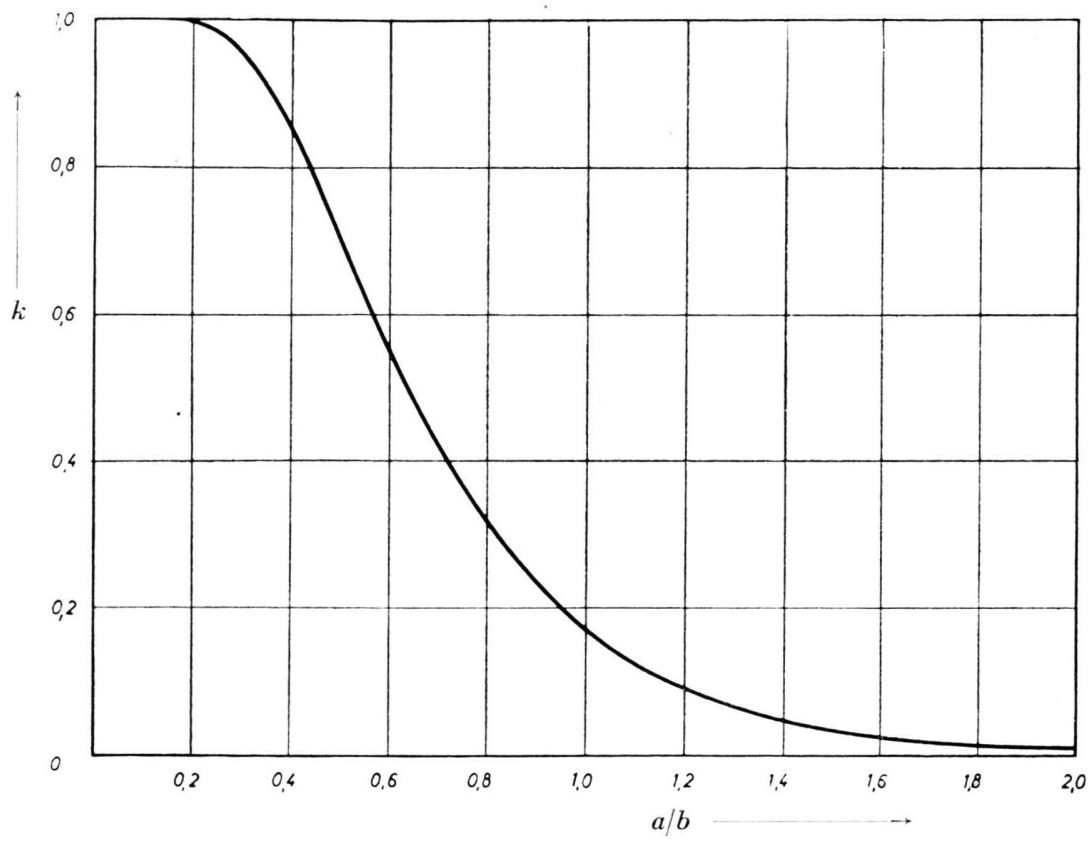

Abb. 4. Zusammenhang zwischen Seitenverhältnis $a / b$ und Modul $k$.

\section{Berechnung der Hall-Spannung einer recht- eckigen Platte}

Die Hald-Spannung $U_{\mathrm{h}}$ ist die Potentialdifferenz zwischen den Punkten $P$ und $Q$ in der $z$-Ebene bzw. den Punkten $+1 / \sqrt{k}$ und $-1 / \sqrt{k}$ in der $\zeta$-Ebene. Da die Stromelektroden Äquipotentiallinien sind, liegen die beiden Punkte $\xi_{1}=+1$ und $\xi_{4}=-1$ auf gleichem Potential. Die Hall-Spannung $U_{\mathrm{h}}$ kann also dargestellt werden als Differenz zweier Linienintegrale über die elektrische Feldstärke $\mathfrak{F}(\xi, \eta)$ entlang der $\xi$-Achse von -1 bis $-1 / \sqrt{ } k$ und von +1 bis $+1 / \sqrt{k}$, also

$$
U_{\mathrm{h}}=\int_{-1}^{-1 / V k}|\xi| \cos \Theta \mathrm{d} \xi-\int_{1}^{1 / 1 / k}|\xi| \cos \Theta \mathrm{d} \xi .
$$

Hierin ist $|\&|$ als Funktion von $\xi$ durch Gl. (8) bestimmt. Durch die Substitution $\xi^{\prime}=-1 /(k \xi)$ kann gezeigt werden, daß

$$
\int_{-1}^{-1 / V k}|\xi| \mathrm{d} \xi=\int_{1 / \gamma k}^{1 / k}|\xi| \mathrm{d} \xi
$$

ist. Für die HaLl-Spannung erhält man daher

$$
U_{\mathrm{h}}=C^{\prime} \cos \Theta\left(Z_{\mathrm{I}}-Z_{\mathrm{II}}\right) \text {, }
$$

wobei die Integrale $Z_{\mathrm{I}}$ und $Z_{\mathrm{II}}$ unter Berücksichtigung der durch die Betragstriche im Integranden festgelegten Vorzeichen lauten:

$$
\begin{aligned}
& Z_{\mathrm{I}}=\int_{1 / \sqrt{k}}^{1 / k}\{(\xi-1)([1 / k]+\xi)\}^{1 / 2-\Theta / \pi\{(\xi+1)([1 / k]-\xi)\}^{1 / 2+\theta / \pi}}, \\
& Z_{\mathrm{II}}=\int_{1}^{1 / \sqrt{k}} \frac{\mathrm{d} \xi}{\{(\xi-1)([1 / k]+\xi)\}^{1 / 2-\Theta / \pi}\{(\xi+1)([1 / k]-\xi)\}^{1 / 2+\Theta / \pi}} .
\end{aligned}
$$

Zur Bestimmung der Konstanten $C^{\prime}$ in Gl. (10) wird der die rechteckige Probe durchfließende Strom $i_{\mathrm{s}}$ in Integralform ausgedrückt. $i_{\mathrm{s}}$ ist das mit der Plattendicke $d$ multiplizierte Integral über die Normalkomponente der Stromdichte i entlang der unteren Stromelektrode $(-1 \leq \xi \leq+1)$

$$
i_{\mathrm{s}}=d \cos \Theta \int_{-1}^{+1}|\mathrm{j}| \mathrm{d} \xi=d \sigma(B) \cos ^{2} \Theta \int_{-1}^{+1}|\xi| \mathrm{d} \xi,
$$

da $|\mathrm{i}|=\sigma(B) \cos \Theta|\mathfrak{F}|$. Aus Gl. (11) wird mit Gl. (8) unter Berücksichtigung der durch die Betragstriche im Integranden festgelegten Vorzeichen

$$
i_{\mathrm{s}}=d \sigma(B) \cos ^{2} \Theta C^{\prime} N
$$


mit

$$
N=\int_{-1}^{+1}\{(1-\xi)([1 / k]+\xi)\}^{1 / 2-\theta / \pi}\{(1+\xi)([1 / k]-\xi)\}^{1 / 2+\theta / \pi} .
$$

Mit Hilfe von Gl. (12) läßt sich die Konstante $C^{\prime}$ in Gl. (10) eliminieren. Der Ausdruck für die HalLSpannung lautet dann

$$
U_{\mathrm{h}}=\underset{d \sigma(B) \cos \Theta}{i_{\mathrm{S}}}{ }^{Z_{\mathrm{I}}-Z_{\mathrm{II}}} .
$$

Erweitert man Gl. (13) mit dem Produkt $R_{\mathrm{h}} B$, so wird mit (1) und (4)

$$
U_{\mathrm{h}}=U_{\mathrm{h} \infty} G(a / b, \Theta)
$$

mit

$$
G(a / b, \Theta)=\underset{\sin \Theta}{1} Z_{\mathrm{I}}-Z_{\mathrm{II}} .
$$

Die HaLL-Spannung $U_{\mathrm{h}}$ einer rechteckigen Halbleiterplatte mit dem Seitenverhältnis $a / b$ und in der Mitte der Längsseiten angebrachten punktförmigen HALLElektroden läßt sich nach Gl. (14) darstellen als Produkt aus der Hald-Spannung $U_{\mathrm{h} \infty}$ des unendlich langen Streifens und einer Geometriefunktion $G(a / b, \Theta)$.

\section{Herleitung analytischer Ausdrücke für die Geometriefunktion $G(a / b, \Theta)$ durch Entwick- lungen}

Eine geschlossene Auswertung der Integrale (10 a), (10 b) und (12 a) ist nicht möglich. Im folgenden werden deshalb Entwicklungen dieser Integrale durchgeführt, die für kleine $\Theta$ und für $\Theta \rightarrow \pi / 2$ auf analytische Ausdrücke für die Geometriefunktion $G(a / b, \Theta)$ führen.

\subsection{Entwicklung von $G(a / b, \Theta)$ für kleine $\Theta$}

3.1.1. Die Integrale $Z_{\mathrm{I}}, Z_{\mathrm{II}}$ und $N$. Die Entwicklung des Nennerintegrals (12 a) für kleine HaLLWinkel ${ }^{6} \Theta$ ergibt für kleine $a / b$

$$
\begin{gathered}
N=2 k K(k)\left\{\begin{array}{cc}
1+\Theta^{2} & 7 S_{3} \\
2 \pi^{2} K(k)
\end{array}\right\} \\
S_{3}=\frac{8}{7} \sum_{n=0}^{\infty} \frac{1}{(2 n+1)^{3}}=1,2021
\end{gathered}
$$

und für große $a / b$

$$
N=2 k K(k)\left\{1+\Theta^{2} \frac{\pi}{4 K(k)}\right\} .
$$

(16 b)

Erweitert man die Integranden der Zählerintegrale $Z_{\mathrm{I}}$ und $Z_{\mathrm{II}}$ mit dem Modul $k$ und führt in (10 a) die Substitution

$$
\lambda=\frac{(\xi+1)(1-k \xi)}{(\xi-1)(1+k \xi)}
$$

und in $(10 \mathrm{~b})$ die reziproke Substitution

$$
\begin{array}{ll}
1 & (\xi+1)(1-k \xi) \\
\lambda & (\xi-1)(1+k \xi)
\end{array}
$$

durch, so erhält man für die in der Geometriefunktion auftretende Differenz der Integrale

$Z_{\mathrm{I}}-Z_{\mathrm{II}}=k \int_{0}^{1} \begin{array}{cc}\lambda-\theta / \pi-\lambda \Theta / \tau & \mathrm{d} \lambda \\ V \lambda & V 4 k(1+\lambda)^{2}+(1-k)^{2}(1-\lambda)^{2}\end{array}$

Die weitere Substitution $\lambda=e^{-2 v}$ führt auf die Form

$$
Z_{\mathrm{I}}-Z_{\mathrm{II}}=2 k \int_{0}^{\infty} \frac{\operatorname{Sin}(2[\Theta / \pi] v)}{V 4 k+(1+k)^{2} \operatorname{Sin}^{2} v} \mathrm{~d} v .
$$

Für kleine $\Theta$ läßt sich $\operatorname{Sin}(2[\Theta / \pi] v)$ in eine TAYLOR-Reihe nach $\Theta / \pi$ entwickeln. Aus der Entwicklung bis zur dritten Potenz in $\Theta / \pi$ folgt dann für $Z_{\mathrm{I}}-Z_{\mathrm{II}}$

$$
\begin{gathered}
Z_{\mathrm{I}}-Z_{\mathrm{II}}=4 k{ }_{\pi}^{\Theta}\left\{D_{1}(k)+\begin{array}{l}
2 \\
3
\end{array}\left(\begin{array}{l}
\Theta \\
\pi
\end{array}\right)^{2} D_{2}(k)\right. \\
D_{1}(k)=\int_{0}^{\infty} \frac{v \mathrm{~d} v}{\sqrt{4} k+(1+k)^{2} \operatorname{\Xi in}^{2} v}
\end{gathered}
$$

mit

und

$$
D_{2}(k)=\int_{0}^{\infty} \frac{v^{3} \mathrm{~d} v}{\sqrt{4} k+(1+k)^{2} \operatorname{Sin}^{2} v} .
$$

Die Integrale (20) und (21) führen für kleine $a / b$ $(a / b \rightarrow 0, k \rightarrow 1)$ und große $a / b(a / b \rightarrow \infty, k \rightarrow 0)$ auf einfache Näherungsausdrücke. Beschränkt man sich bei der Entwicklung für kleine $a / b$ im Hinblick auf die $k$-Abhängigkeit der Geometriefunktion $G(a / b, \Theta)$ auf die erste Näherung, so kann man setzen ${ }^{8}$

$$
\begin{aligned}
& D_{1}(k)=D_{1}(1)=\frac{1}{2} \int_{0}^{\infty} \frac{v \mathrm{~d} v}{\operatorname{So} \mathfrak{j} v} \\
& =\sum_{n=0}^{\infty} \frac{(-1)^{n}}{(2 n+1)^{2}}=T_{2}=0,91597 \\
& \text { und } \quad \begin{aligned}
D_{2}(k) & =D_{2}(1)=\frac{1}{2} \int_{0}^{\infty} \frac{v^{3} \mathrm{~d} v}{\mathfrak{E} \mathfrak{o} i} \\
& =6 \sum_{n=0}^{\infty} \frac{(-1)^{n}}{(2 n+1)^{4}}=6 T_{4}=6 \cdot 0,98894 .
\end{aligned}
\end{aligned}
$$

\footnotetext{
${ }^{8}$ F. TöLkE, Praktische Funktionenlehre, Springer-Verlag, Berlin 1950, S. $364 \mathrm{f}$.
} 
Für große $a / b(k \rightarrow 0)$ reicht es nicht aus, sich auf das Glied $D_{1}(k)=D_{1}(0)$ zu beschränken. Um die $k$-Abhängigkeit der Geometriefunktion bis zur zweiten Näherung zu erhalten, wird im Anhang eine Entwicklung von $D_{1}(k)$ für kleine $k$ bis zum zweiten Glied durchgeführt. Es ergibt sich:

$D_{1}(k)=\left(\pi^{2} / 4\right)\left\{1-\left(8 / \pi^{2}\right) \sqrt{k}(1-[2 / 9] k)\right\}$.

Da das Integral (21) für $D_{2}(k)$ in Gl. (19) mit $(\Theta / \pi)^{2}$ multiplikativ behaftet ist, $\Theta / \pi$ aber voraussetzungsgemäß klein ist, so kann man für große $a / b$ setzen

$$
\begin{aligned}
D_{2}(k)=D_{2}(0) & =\int_{0}^{\infty} v_{\operatorname{Sin} \nu} \mathrm{d} v \\
& =12 \sum_{n=0}^{\infty} \frac{1}{(2 n+1)^{4}}=12 \frac{\pi^{4}}{96} \quad \frac{\pi^{4}}{8} .
\end{aligned}
$$

Zusammenfassend gilt damit für den Zähler $Z_{\mathrm{I}}-Z_{\mathrm{II}}$ der Geometriefunktion $G(a / b, \Theta)$ für kleine $a / b$

$$
Z_{\mathrm{I}}-Z_{\mathrm{II}}={ }_{\pi}^{4} T_{2} k \Theta\left\{1+\begin{array}{cc}
4 & T_{4} \\
\pi^{2} & T_{2}
\end{array} \Theta^{2}\right\}
$$

und für große $a / b$

$Z_{\mathrm{I}}-Z_{\mathrm{II}}=\pi k \Theta\left\{1-\frac{8}{\pi^{2}} \sqrt{ } k\left(1-\frac{2}{9} k\right)+\frac{\Theta^{2}}{3}\right\}$.

3.1.2. Analytische Ausdrücke für $G(a / b, \Theta)$ für kleine $\Theta$ und deren Gültigkeit. Durch Einsetzen von (16 a u. b) sowie (23 a u. b) in Gl. (15) ergeben sich unter gleichzeitiger Entwicklung von

$$
\sin \Theta=\Theta\left(1-\left[\Theta^{2} / 6\right]\right)
$$

für die Geometriefunktion $G(a / b, \Theta)$ bei kleinen HaLL-Winkeln $\Theta$ die Ausdrücke:

und

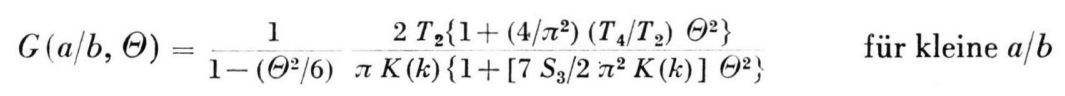

$$
G(a / b, \Theta)=\begin{array}{ccc}
1 & \pi\left\{1-\left(8 / \pi^{2}\right) \sqrt{ } k(1-[2 / 9] k)+\Theta^{2} / 3\right\} & \text { für große } a / b . \\
1-\left(\Theta^{2} / 6\right) & 2 K(k)\left\{1+[\pi / 4 K(k)] \Theta^{2}\right\} &
\end{array}
$$

Mit ( 7 a) und dem Näherungsausdruck des vollständigen elliptischen Integrals $K(k)$ für $k \rightarrow 1$, also für $a / b \rightarrow 0\left(\right.$ s. Anm. $\left.{ }^{7}\right)$ folgt aus $(24 \mathrm{a})$

$$
G(a / b, \Theta)=\frac{8}{\pi^{2}} T_{2} \frac{a}{b}\left\{1+\frac{\Theta^{2}}{6}\left(1+\frac{24 T_{4}}{\pi^{2} T_{2}}-\frac{84 S_{3}}{\pi^{3}} \frac{a}{b}\right)\right\} .
$$

Dieser Entwicklungsausdruck gilt mit einem Fehler bis zu ca. $1 \%$ in dem Wertebereich $0 \leq a / b \leq 0,35$ und $0 \leq \Theta \leq 0,45$. Entsprechend erhält man aus $(24 \mathrm{~b})$ mit $(7 \mathrm{~b})$ und dem Näherungsausdruck des vollständigen elliptischen Integrals $K(k)$ für $k \rightarrow 0$, also $a / b \rightarrow \infty$ (s. Anm. ${ }^{7}$ ),

$$
G(a / b, \Theta)=1-\frac{16}{\pi^{2}} \exp \left\{-\frac{\pi}{2} \frac{a}{b}\right\}\left(1-\frac{8}{9} \exp \left\{-\pi \frac{a}{b}\right\}\right)\left(1-\frac{\Theta^{2}}{3}\right)
$$

mit dem Gültigkeitsbereich $1,0 \leq a / b \leq \infty$ und $\quad 0 \leq \Theta \leq 0,45$.

\subsection{Entwicklung von $G(a / b, \Theta)$ für $\Theta \rightarrow \pi / 2$}

3.2.1. Die Integrale $Z_{\mathrm{I}}, Z_{\mathrm{II}}$ und $N$. Wir beginnen mit der Entwicklung des Integrals $Z_{\mathrm{I}}$ Gl. (10 a). Als Entwicklungsgröße wird $\varepsilon=1 / 2-\Theta / \pi$ gewählt. Integral $Z_{\mathrm{I}}$ lautet dann:

$$
Z_{\mathrm{I}}=\int_{1 / \sqrt{ } k}^{1 / k} \frac{\mathrm{d} \xi}{\{(\xi-1)([1 / k]+\xi)\} \varepsilon\{(\xi+1)([1 / k]-\xi)\}^{1-\varepsilon}} .
$$

Der Integrand hat im Integrationsbereich eine Singularität bei $\xi=1 / k$. Das Integral über diese Singularität divergiert für $\varepsilon \rightarrow 0$, d. h. für $\Theta \rightarrow \pi / 2$. Da eine Entwicklung des Integrals für $\varepsilon \rightarrow 0$ angestrebt wird, das Verhalten von $Z_{\mathrm{I}}$ aber entscheidend durch die Singularität bei $\xi=1 / k$ beherrscht wird, spalten wir einen Term $Z_{\mathrm{I}}^{(0)}(\varepsilon) \mathrm{ab}$, der diese Singularität in geschlossen integrierbarer Form enthält: 


$$
Z_{\mathrm{I}}=Z_{\mathrm{I}}^{(\mathbf{0})}(\varepsilon)+Z_{\mathrm{I}}^{(1)}(\varepsilon) \quad \text { mit } \quad Z_{\mathrm{I}}{ }^{(0)}(\varepsilon)=p \int_{1 / V k}^{1 / k}([1 / k]-\xi)^{1-\varepsilon}
$$

und

$$
Z_{\mathrm{I}}{ }^{(1)}(\varepsilon)=\int_{1 / V k}^{1 / k}\left[\left\{\begin{array}{c}
1 \\
\{(\xi-1)([1 / k]+\xi)\}^{\varepsilon}\{(\xi+1)([1 / k]-\xi)\}^{1-\varepsilon}- \\
([1 / k]-\xi)^{1-\varepsilon}
\end{array}\right] \mathrm{d} \xi .\right.
$$

Die Konstante $p$ wird so bestimmt, daß für $\xi=1 / k$ der Zähler des Integranden von $Z_{I}{ }^{(1)}(\varepsilon)$ verschwindet, und damit der Integrand von $Z_{\mathrm{I}}{ }^{(1)}(\varepsilon)$ bei $\xi=1 / k$ regulär wird. Diese Bedingung liefert

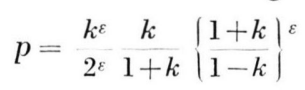

und daher für

$$
\begin{aligned}
Z_{\mathrm{I}}^{(0)}(\varepsilon)=p \int_{1 / V^{k}}^{1 / k} \frac{\mathrm{d} \xi}{([1 / k]-\xi)^{1-\varepsilon}} \\
=\frac{1}{\varepsilon} \frac{k}{1+k}\left\{\frac{(1+k)(1-V k)}{2(1-k)}\right\}^{\varepsilon}
\end{aligned}
$$

oder für kleine $\varepsilon$ entwickelt

$$
Z_{\mathrm{I}}^{(0)}(\varepsilon)=\frac{k}{1+k}\left\{\frac{1}{\varepsilon}+\ln \frac{(1+k)(1-V k)}{2(1-k)}\right\} .
$$

$Z_{\mathrm{I}}^{(1)}(\varepsilon)$ kann kein Entwicklungsglied mit $1 / \varepsilon$ mehr enthalten. Das nächst höhere Glied in der Entwicklung nach Potenzen von $\varepsilon$ ist das von $\varepsilon$ freie Glied. Das Integral $Z_{\mathrm{I}}{ }^{(1)}(\varepsilon)$ liefert zu diesem Glied den Beitrag

$$
\begin{aligned}
Z_{\mathrm{I}}^{(1)}(\varepsilon & =0) \\
& =\int_{1 / V k}^{1 / k}\left[\frac{1}{(\xi+1)([1 / k]-\xi)}-\frac{k}{1+k} \frac{1}{([1 / k]-\xi)}\right] \mathrm{d} \xi .
\end{aligned}
$$

Nach Partialbruchzerlegung des ersten Terms in diesem Integral erhält man

$$
Z_{\mathrm{I}}^{(1)}(\varepsilon=0)=\frac{k}{1+k} \ln \frac{(1+k)}{\sqrt{k}(1+\sqrt{k})} .
$$

Die Summe aus der Entwicklung von $Z_{\mathrm{I}}{ }^{(0)}(\varepsilon)$ und dem Ausdruck $Z_{\mathrm{I}}{ }^{(1)}(\varepsilon=0)$ liefert nach $(27 \mathrm{a})$ die Entwicklung des Integrals $Z_{\mathrm{I}}$ bis zum zweiten Glied, also

$$
Z_{\mathrm{I}}=\frac{k}{1+k} \frac{1}{\varepsilon}\left\{1+\varepsilon \ln \frac{(1+k)^{2}}{2 \sqrt{k(1+V k)^{2}}}\right\} .
$$

Das Integral $Z_{\mathrm{II}}$ lautet nach Einführung der Entwicklungsgröße $\varepsilon$

$$
Z_{\mathrm{II}}=\int_{1}^{1 / \sqrt{k}} \frac{\mathrm{d} \xi}{\{(\xi-1)([1 / k]+\xi)\}^{\varepsilon}\{(\xi+1)([1 / k]-\xi)\}^{1-\varepsilon}} .
$$

Der Integrand besitzt im Integrationsbereich eine Singularität bei $\xi=+1$. Diese Singularität ist inte- grabel und ergibt für $\varepsilon \rightarrow 0$ einen endlichen von $\varepsilon$ unabhängigen Beitrag zum Gesamtintegral. Das erste Glied in einer Entwicklung nach $\varepsilon$ ist daher das von $\varepsilon$ freie Glied, also

$Z_{\mathrm{II}}(\varepsilon=0)=\int_{1}^{1 / V^{k}} \frac{\mathrm{d} \xi}{(\xi+1)([1 / k]-\xi)} \frac{k}{1+k} \ln \frac{(1+\sqrt{k})^{2}}{2 \sqrt{k}}$.

Als Entwicklung der in $G(a / b, \Theta)$ Gl. (15) auftretenden Differenz $Z_{\mathrm{I}}-Z_{\mathrm{II}}$ erhält man schließlich mit (28) und (30)

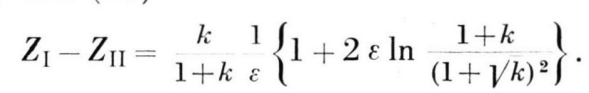

Die Entwicklung des Nennerintegrals (12 a) liefert für große HaLL-Winkel $\Theta \rightarrow \pi / 2$ in Analogie $^{6} \mathrm{zu}$ $Z_{\mathrm{I}}$ und $Z_{\mathrm{II}}$

$$
N=\frac{k}{1+k} \quad \frac{1}{\varepsilon}\left\{1+2 \varepsilon \ln \frac{1+k}{1-k}\right\} .
$$

3.2.2. Analytischer Ausdruck für $G(a / b, \Theta)$ bei großen Hald-Winkeln und dessen Gültigkeit. Durch Einsetzen der Entwicklungen (31) und (32) in Gl. (15) folgt für die Geometriefunktion $G(a / b, \Theta)$ bei gleichzeitiger Entwicklung von $\sin \Theta=\cos \pi \varepsilon$ $=1 \mp$...

$$
G(a / b, \Theta)=\frac{1+2 \varepsilon \ln \left\{[1+k] /\left[(1+\sqrt{k})^{2}\right]\right\}}{1+2 \varepsilon \ln \{(1+k) /(1-k)\}} .
$$

In Ausdruck (33) für $G(a / b, \Theta)$ sind Zähler und Nenner Entwicklungen nach $\varepsilon$ bis zum linearen Glied. Um den Wertebereich der Variablen $a / b$ und $\Theta$ angeben zu können, in dem Gl. (33) mit einem Fehler kleiner ca. $1 \%$ gilt, so muß die Gültigkeit der Entwicklungen für den Zähler, d. h. für die Integrale $Z_{\mathrm{I}}$ und $Z_{\mathrm{II}}$, sowie für den Nenner, d. h. für das Integral $N$ und $\sin \Theta$ getrennt bestimmt werden. Wenn in $Z_{\mathrm{I}}$ das lineare Glied

$$
\varepsilon\left|\ln \frac{(1+k)^{2}}{2 \sqrt{k}(1+\sqrt{k})^{2}}\right|<0,1
$$

bleibt, wird das quadratische Glied in $\varepsilon$ von der Größenordnung 0,01 sein ${ }^{9}$. Diese Bedingung wird

\footnotetext{
$9 \mathrm{Da} \beta$ diese Abschätzung des Gültigkeitsbereichs erlaubt ist, zeigt der Vergleich der durch Entwicklung gewonnenen Ergebnisse mit der numerischen Auswertung von (15) mit $(10 \mathrm{a}),(10 \mathrm{~b})$ und $(12 \mathrm{a})$.
} 
oberhalb der im $\Theta-a / b$-Diagramm (Abb. 5) aus zwei Ästen bestehenden Kurve 1 erfüllt. Für die Gültigkeit von $Z_{\mathrm{II}}$ gilt in gleicher Weise

$$
\varepsilon \ln \frac{\left(1+{\sqrt{k})^{2}}_{2} V^{k}\right.}{2}<0,1
$$

(Gültigkeitsbereich oberhalb der Kurve 2 in Abb.5). Die Bedingung

$$
\varepsilon \ln \frac{1+k}{1-k}<0,1
$$

führt auf den Gültigkeitsbereich der Entwicklung des Nennerintegrals $N$ oberhalb der Kurve 3 in Abb. 5. Für die Entwicklung von $\sin \Theta$ gilt schließlich $\pi \varepsilon<0,14$ (Bereich oberhalb der in Abb. 5 zur Abszisse parallelen Geraden 4). Für die Gültigkeit

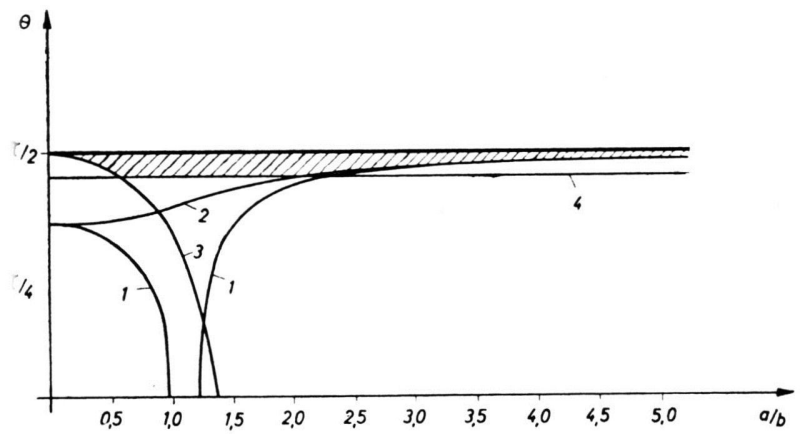

Abb. 5. Gültigkeitsbereich der Entwicklung für große Magnetfelder $(\Theta \rightarrow \pi / 2)$.

des Ausdrucks (33) müssen alle vier Bedingungen gleichzeitig erfüllt sein. Als Gültigkeitsbereich für Gl. (33) resultiert daher das in Abb. 5 schraffierte Gebiet.

Für die Geometriefunktion $G(a / b, \Theta)$ erhält man somit in dem vorangehend abgegrenzten Werte- bereich mit $\pi \varepsilon=\operatorname{cotg} \Theta$

$$
G(a / b, \Theta)=1-\frac{2}{\pi} \ln \frac{1+V^{k}}{1-V^{k}} \cdot \operatorname{cotg} \Theta .
$$

\section{Zusammenfassung und Diskussion der Ergebnisse}

Die Lösung des Randwertproblems einer rechteckigen, stromdurchflossenen Platte im transversalen Magnetfeld führt auf einen Integralausdruck für die zwischen den Punkten $P$ und $Q$ in der Mitte der Platte auftretende HaLL-Spannung $U_{\mathrm{h}}$ (Abb. 1). Danach läßt sich die HaLL-Spannung $U_{\mathrm{h}}$ darstellen als Produkt der Hall-Spannung $U_{\mathrm{h} \infty}$ des unendlich langen Streifens und einer Geometriefunktion $G(a / b, \Theta)$, die vom Seitenverhältnis $a / b$ der rechteckigen Platte und vom HaLL-Winkel $\Theta$ abhängt.

Die Entwicklung der Integrale in $G(a / b, \Theta)$ liefert einfache analytische Ausdrücke, die das Verhalten der Geometriefunktion für kleine und große HaLL-Winkel beschreiben. In Tab. 1 sind die in Abschnitt 3 gewonnenen Entwicklungsausdrücke für $G(a / b, \Theta)$ zusammengestellt.

Die Größe $k$ in (34) ist durch die transzendente Gl. (7) als Funktion des Seitenverhältnisses $a / b$ bestimmt. Die Ausdrücke (35) und (36) erhält man aus (34) mit Hilfe der Entwicklungen (7a) und (7 b) .

In Abb. 6 sind in einem $\Theta-a / b$-Diagramm die Gültigkeitsbereiche für die angegebenen Entwicklungen schraffiert eingezeichnet. Der Wertevorrat zerfällt danach in drei nicht zusammenhängende $\mathrm{Be}$ -

\begin{tabular}{|c|c|c|}
\hline $\begin{array}{l}\text { Seitenverhältnis } \\
a / b\end{array}$ & kleine HALL$_{\text {-Winkel }} \Theta$ & große HALL-Winkel $\Theta$ \\
\hline beliebig & & $1-\frac{2}{\pi} \ln \frac{1+\sqrt{ } k}{1-\sqrt{ } k} \operatorname{cotg} \Theta$ \\
\hline klein & $\frac{8}{\pi^{2}} T_{2} \frac{a}{b}\left\{1+\frac{\Theta^{2}}{6}\left(1+{ }_{\pi^{2} T_{2}}^{24} T_{4}-\frac{84 S_{3}}{\pi^{3}} \frac{a}{b}\right)\right\} \quad$ (25) & $1-\left(\frac{b}{a}-\frac{2 \ln 2}{\pi}-\frac{4}{\pi} \exp \left\{-\frac{\pi}{2} \frac{b}{a}\right\}\right) \operatorname{cotg} \Theta$ \\
\hline groß & $1-\frac{16}{\pi^{2}} \exp \left\{-\frac{\pi}{2} \frac{a}{b}\right\}\left(1-\frac{8}{9} \exp \left\{-\pi \frac{a}{b}\right\}\right)\left(1-\frac{\Theta^{2}}{3}\right)$ & $1-\frac{8}{\pi} \exp \left\{-\frac{\pi}{2} \frac{a}{b}\right\} \operatorname{cotg} \Theta$ \\
\hline
\end{tabular}
reiche I, II und III. I ist der Gültigkeitsbereich für Gl. (25), II der Gültigkeitsbereich für (26) und

Tab. 1. 


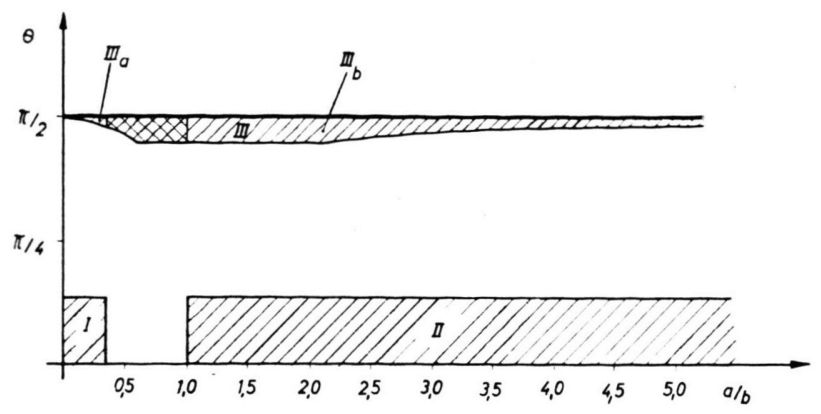

Abb. 6. Gültigkeitsbereiche der analytischen Ausdrücke für $G(a / b, \Theta)$.

schließlich III der Gültigkeitsbereich für Gl. (34) mit den Unterbereichen III a und III b für (35) und (36). Der Gültigkeitsbereich der angeführten Entwicklungen ist dadurch definiert, daß für den Wertevorrat der einzelnen Bereiche der Fehler kleiner ca. $1 \%$ bleibt. Der durch die schraffierten Bereiche nicht überdeckte Wertevorrat im $\Theta-a / b$-Diagramm wurde durch numerische Auswertung der Integrale (10 a), $(10 \mathrm{~b})$ und $(12 \mathrm{a})$ erschlossen.

Abb. 7 zeigt $G(a / b, \Theta)$ als Funktion von $a / b$ mit $\Theta$ als Parameter. Mit $\Theta \rightarrow 0$ strebt die Geometrie-

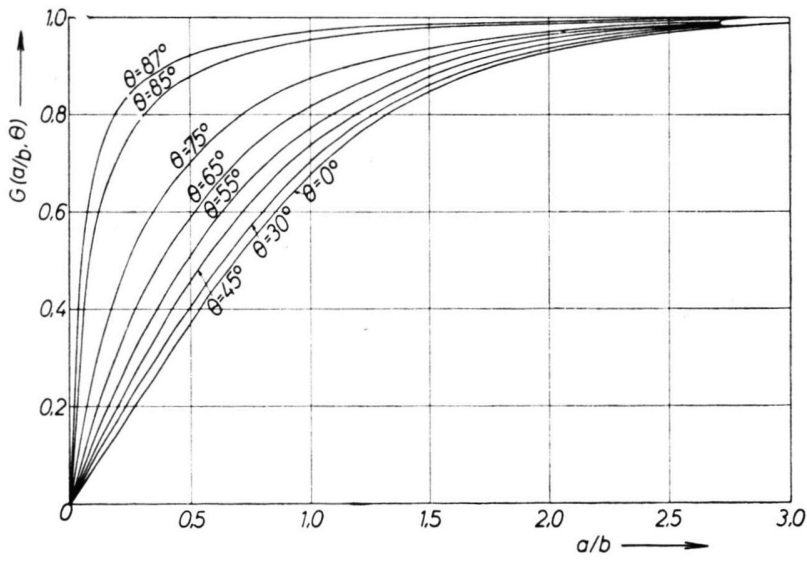

Abb. 7. Geometriefunktion $G(a / b, \Theta)=U_{\mathrm{h}} / U_{\mathrm{h}_{\infty}}$ als Funktion des Seitenverhältnisses $a / b$ mit HALL-Winkeln $\Theta$ als Parameter.

funktion gegen die Grenzkurve $G(a / b, 0)$, die sich für kleine $a / b$ nach (25) wie eine Nullpunktsgerade mit dem Anstieg $\left(8 / \pi^{2}\right) T_{2}$ verhält und für große $a / b$ nach (26) exponentiell in die im Abstand 1 zur Abszisse parallele Gerade einmündet. Für alle $\Theta \neq 0$ wird $G(a / b, \Theta)$ durch Kurven dargestellt, die oberhalb dieser Grenzkurve liegen. Für $a / b \rightarrow \infty$ geht

10 Für die numerische Auswertung der Integrale (10a), (10b) und (12a) danken wir den Herren H. Hofrmans und G. ZEGLIN vom Mathematischen Institut der SiemensSchuckertwerke AG., Erlangen. daher $G(a / b, \Theta)$ für alle $\Theta$ gegen 1 , d. h. die HaLLSpannung $U_{\mathrm{h}}$ wird identisch mit der HaLL-Spannung $U_{\mathrm{h} \infty}$ des unendlich langen Streifens. Bis zu einem HaLL-Winkel $\Theta$ von ca. $30^{\circ}$ werden die Kurven $G(a / b, \Theta)$ durch (25) und (26) beschrieben, für $\Theta \rightarrow \pi / 2$ (d.h. im Gültigkeitsbereich III in Abb. 6) durch (34). In dem Zwischengebiet, in dem die Gültigkeit der analytischen Entwicklungsausdrücke versagt, wurde $G(a / b, \Theta)$ durch numerische Auswertung der Integrale (10a), (10b) und (12a) bestimmt ${ }^{10}$.

Für kleine HaLL-Winkel $\Theta$, d. h. für kleine Magnetfelder $B$ erhält man nach (14) unter Berücksichtigung von (1), (4) und (25) bzw. (26) für die HaLL-Spannung $U_{\mathrm{h}}$ eine Entwicklung nach $B$ bis zur dritten Potenz. In dieser Entwicklung ist das lineare Glied in $B$ mit einem Koeffizienten behaftet, der für alle endlichen Seitenverhältnisse $a / b$ kleiner ist als der Proportionalitätsfaktor $R_{\mathrm{h}} i_{\mathrm{s}} / d$ zwischen der HaLL-Spannung des unendlich langen Streifens $U_{\mathrm{h} \infty}$ und $B$. Von den elektrischen Daten des Halbleitermaterials enthält dieser Koeffizient nur die HaLLKonstante $R_{\mathrm{h}}$. In den Entwicklungskoeffizienten des Gliedes $B^{3}$ geht dagegen neben $R_{\mathrm{h}}$ auch die Leitfähigkeit $\sigma(B)$ ein. $\sigma(B)$ kann in dem Koeffizienten von $B^{3}$ durch $\sigma(0)$ ersetzt werden. Die Abnahme der Leitfähigkeit $\sigma$ mit dem Magnetfeld ist nämlich für kleine Magnetfelder quadratisch ${ }^{11}$ in $B$ und wirkt sich daher erst im Entwicklungskoeffizienten des Gliedes 5. Ordnung in $B$ aus.

Für große Magnetfelder $(\Theta \rightarrow \pi / 2)$ wird das asymptotische Verhalten der HaLL-Spannung $U_{\mathrm{h}}$ nach (14) mit (1), (4) und (34) beschrieben durch

$$
U_{\mathrm{h}}=\frac{R_{\mathrm{h}}}{d} i_{\mathrm{s}} B-\frac{2}{\pi} \frac{i_{\mathrm{S}}}{d \sigma(B)} \cdot \ln \frac{1+\sqrt{k}}{1-\sqrt{k}} .
$$

Nach (35) ist die HaLl-Spannung $U_{\mathrm{h}}$ für große Magnetfelder gleich der HaLL-Spannung des unendlich langen Streifens vermindert um ein Glied, das nur wegen $\sigma(b)$ magnetfeldabhängig ist. Die Leitfähigkeit $\sigma(B)$ nimmt für große Magnetfelder nur schwach mit wachsendem $B$ ab, so daß die HaLLSpannung einer rechteckigen Platte mit endlichem Seitenverhältnis $a / b$ wegen des ersten Termes in Gl. (37) praktisch linear mit $B$ wächst. Da die

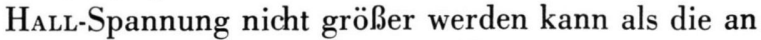
die Probe angelegte Spannung $U$, ist es interessant, 11 H. FröHцich, Elektronentheorie der Metalle, Springer-Verlag, Berlin 1936, S. 232 ff. 
in diesem Zusammenhang das asymptotische Verhalten von $U$ anzugeben. Für große Magnetfelder $B$ wird $U$ beschrieben durch ${ }^{6}$

$$
U=\frac{R_{\mathrm{h}}}{d} i_{\mathrm{s}} B+\frac{2}{\pi} \frac{i_{\mathrm{s}}}{d \sigma(B)} \ln \frac{1-k}{2 \sqrt{k}},
$$

so daß man für die Differenz zwischen Plattenspannung $U$ und HaLL-Spannung $U_{\mathrm{h}}$ den für alle $k(0 \leq k \leq 1)$ positiven Ausdruck

$$
U-U_{\mathrm{h}}=\frac{2}{\pi} \frac{i_{\mathrm{s}}}{d \sigma(B)} \ln \frac{(1+\sqrt{k})^{2}}{2 \sqrt{k}}
$$

erhält oder mit Hilfe der Entwicklungen ( 7 a) und (7 b) für $k \rightarrow 0$ und $k \rightarrow 1$

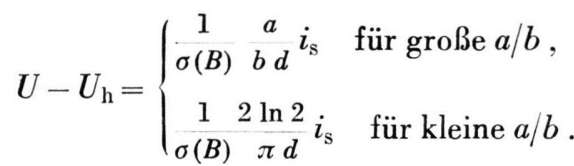

Die HaLL-Spannung $U_{\mathrm{h}}$ wächst zwar für $B \rightarrow \infty$ linear mit $B$ an, bleibt aber stets um den durch (39) angegebenen, für große Magnetfelder annähernd konstanten Betrag kleiner als die an die Platte angelegte Spannung $U$.

\section{Anhang}

Entwicklung von $D_{1}(k)$ für $k \rightarrow 0$

Aus dem Integral (20) für $D_{1}(k)$ wird $(1+k)$ ausgeklammert. Man erhält dann

$$
\begin{aligned}
D_{1}(k) & =\int_{0}^{\infty} \frac{v \mathrm{~d} v}{\sqrt{4} k+(1+k)^{2} \operatorname{\Xi in}^{2} v} \\
& =\frac{1}{1+k} \int_{0}^{\infty} \frac{v \mathrm{~d} v}{\sqrt{x^{2}+\operatorname{Sin}^{2} v}} \quad \frac{1}{1+k} f(x)
\end{aligned}
$$

mit $\varkappa=2 \sqrt{k} /(1+k)$. Mit $k \rightarrow 0$ geht auch $\varkappa$ gegen Null. Man kann sich daher zunächst auf eine Entwicklung von $f(\varkappa)$ für kleine $\varkappa$ beschränken. Hierzu wird der Integrationsbereich des Integrals $f(\varkappa)$ in der folgenden Weise aufgespalten:

$$
f(x)=\int_{0}^{o} \frac{v \mathrm{~d} v}{\sqrt{x^{2}+\operatorname{\Xi in}^{2} v}}+\int_{\varrho}^{\infty} \frac{v \mathrm{~d} v}{V x^{2}+\Xi_{\operatorname{in}^{2}} v}
$$

Wird nun $\varrho$ so gewählt, daß stets $\varrho \gg \varkappa$ ist, gleichzeitig aber $\varrho$ mit $\varkappa \rightarrow 0$ gegen Null geht, so kann man im ersten Integral von (41)

$$
\operatorname{Sin} \nu=v+\frac{v^{3}}{6} \quad \text { oder } \operatorname{Sin}^{2} v=v^{2}+\begin{aligned}
& v^{4} \\
& 3
\end{aligned}
$$

setzen und im zweiten Integral

$$
\begin{aligned}
\sqrt{x^{2}+\operatorname{Sin}^{2} v} & =\operatorname{Sin} v \sqrt{1+\operatorname{Sin}^{2} v} \\
& =\operatorname{Sin} v\left(1+\begin{array}{cc}
x^{2} & 1 \\
2 & \operatorname{Sin}^{2} v
\end{array}\right) .
\end{aligned}
$$

Damit ergibt sich für $f(\varkappa)$

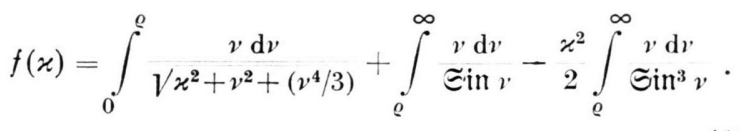

Mit Hilfe der Substitution $v^{2}=\tau$ erhält man für das erste Integral

$\underset{2}{\sqrt{3}} \int_{0}^{\rho^{2}} \frac{\mathrm{d} \tau}{V \tau^{2}+3 \tau+3 \varkappa^{2}} \stackrel{\sqrt{3}}{2} \ln \left\{\begin{array}{c}1+\frac{2}{3}\left(\varrho^{2}+\sqrt{ } \varrho^{4}+3 \varrho^{2}+3 \varkappa^{2}\right) \\ 1+(2 / \sqrt{ }) \varkappa\end{array}\right\}$

oder bis zum Glied dritter Ordnung in $\varrho$ und $\varkappa$ entwickelt:

$\int_{0}^{0} \begin{gathered}v \mathrm{~d} v \\ V \varkappa^{2}+v^{2}+\left(v^{4} / 3\right)\end{gathered}=\varrho-\varkappa+\begin{array}{ccc}1 & \varkappa^{2} & 4 \\ 2 & \varrho & 9\end{array} \varkappa^{2}+\frac{1}{4} \varkappa^{2} \varrho-\begin{gathered}\varrho^{3} \\ 18\end{gathered}$

Das zweite Integral in Gl. (42) kann man in zwei Integrale aufspalten

$\int_{\varrho}^{\infty} \begin{gathered}v \mathrm{~d} v \\ \operatorname{Sin} v\end{gathered}=\int_{0}^{\infty} \frac{v \mathrm{~d} v}{\operatorname{Sin} v}-\int_{0}^{\varrho} \begin{array}{cc}v \mathrm{~d} v & \pi^{2} \\ v\left(1+\left[v^{2} / 6\right]\right) & 4\end{array}-\int_{0}^{\rho}\left(1-\begin{array}{c}v^{2} \\ 6\end{array}\right) \mathrm{d} v$

oder

$$
\int_{\varrho}^{\infty} \begin{array}{ll}
v \mathrm{~d} v & \pi^{2} \\
\operatorname{Sin} v & 4
\end{array}-\varrho+\frac{\varrho^{3}}{18} .
$$

Durch partielle Integration des dritten Integrals in (42) ergibt sich

$$
\int_{\varrho}^{\infty} \begin{array}{ll}
v \mathrm{~d} v & \varrho \operatorname{Eoj} \varrho \\
\operatorname{Sin}^{3} v & 2 \operatorname{Sin}^{2} \varrho
\end{array}+\begin{array}{cc}
1 & 1 \\
2 \operatorname{\Xi in} \varrho & 2
\end{array} \int_{\varrho}^{\infty} \begin{gathered}
v \mathrm{~d} v \\
\operatorname{\Xi in} v
\end{gathered} .
$$

Unter Benutzung von (44) und nach Entwicklung des ausintegrierten Anteils nach $\varrho$ erhält man

$$
\int_{\varrho}^{\infty} \begin{array}{ccc}
v \mathrm{~d} v & 1 & \pi^{2} \\
\operatorname{Sin}^{3} v & \varrho & 8
\end{array}+\frac{\varrho}{2} .
$$

Setzt man (43), (44) und (45) in (42) ein, so lautet die Entwicklung von (41) für kleine $\varkappa$ bis zum dritten Glied

$$
f(\varkappa)=\frac{\pi^{2}}{4}-\varkappa+\frac{\pi^{2}}{16} \varkappa^{2}-4 x^{3} .
$$

Die durch Aufspalten des Integrationsbereichs eingeführte Rechengröße $\varrho$ fällt also wieder heraus. Der Übergang von $f(\varkappa)$ auf $D_{1}(k)$ liefert schließlich die unter 3.1.1. angegebene Entwicklung

$$
D_{1}(k)=\frac{\pi^{2}}{4}-2 k^{1 / 2}+\frac{4}{9} k^{3 / 2}=\frac{\pi^{2}}{4}\left\{1-\frac{8}{\pi^{2}} v k\left(1-\frac{2}{9} k\right)\right\} .
$$

